\title{
Parameterization of entrainment in a sheared convective boundary layer using a first-order jump model
}

\author{
Si-Wan Kim • Soon-Ung Park • David Pino • \\ Jordi Vilà-Guerau de Arellano
}

Received: 20 December 2004 / Accepted: 16 February 2006 /

Published online: 5 June 2006

(C) Springer Science+Business Media, Inc. 2006

\begin{abstract}
Basic entrainment equations applicable to the sheared convective boundary layer (CBL) are derived by assuming an inversion layer with a finite depth, i.e., the first-order jump model. Large-eddy simulation data are used to determine the constants involved in the parameterizations of the entrainment equations. Based on the integrated turbulent kinetic energy budget from surface to the top of the CBL, the resulting entrainment heat flux normalized by surface heat flux is a function of the inversion layer depth, the velocity jumps across the inversion layer, the friction velocity, and the convection velocity. The developed first-order jump model is tested against large-eddy simulation data of two independent cases with different inversion strengths. In both cases, the model reproduces quite reasonably the evolution of the CBL height, virtual potential temperature, and velocity components in the mixed layer and in the inversion layer.
\end{abstract}

Keywords Entrainment heat flux · First-order jump model · Inversion layer depth · Large-eddy simulation $\cdot$ Sheared convective boundary layer

The part of this work was done when the first author visited at NCAR.

S.-W. Kim $(\bowtie)$

Chemical Sciences Division, Earth System Research Laboratory, National Oceanic and Atmospheric Administration, 325, Broadway, R/CSD4, Boulder, CO 80305, USA e-mail: siwan.kim@noaa.gov

\section{S.-W. Kim}

Cooperative Institute for Research in Environmental Sciences, University of Colorado, Boulder, CO, USA

S.-U. Park

School of Earth and Environmental Sciences, Seoul National University, Seoul, Korea

D. Pino

Applied Physics Department, Technical University of Catalonia and Institute for Space Studies of Catalonia, Barcelona, Spain

J. Vilà-Guerau de Arellano

Meteorology and Air Quality Section, Wageningen University, Wageningen, The Netherlands 


\section{Introduction}

In order to represent subgrid processes in the convective boundary layer (CBL), large-scale atmospheric models have adopted the mixed-layer model (Deardorff 1972; Suarez et al. 1983; Ayotte et al. 1996). The key process in the mixed-layer model is the entrainment parameterization. The entrainment parameterization used in these models should be able to represent the entrainment flux for both pure buoyancy and sheared environments. The entrainment heat flux and temperature jump across the inversion layer (or entrainment zone) determine the entrainment rate that is used to predict the mixed-layer height and diagnose the entrainment momentum fluxes. Much work has been done for the purely buoyancy-driven CBL and thus the uncertainties for the entrainment process are relatively small compared to the sheared CBL. The representation of entrainment in the purely buoyancy-driven CBL has been studied using zero-order jump models, first-order jump models, and "realistic" models. The zeroorder jump model represents the inversion layer as an infinitesimally thin interface (Tennekes 1973), while the first-order jump model treats the inversion layer with a finite depth (Betts 1973). In the first-order jump model, a linear variation of variables with height in the inversion layer is assumed. Realistic models consider the variation of variables with height in the inversion layer with a high order polynomial function in order to incorporate the curvature of the profiles (Deardorff 1979; Fedorovich and Mironov 1995).

The influence of shear on the entrainment flux has mostly been based on the zero-order jump model (e.g., Zeman and Tennekess 1977; Pino et al. 2003). The finite thickness of the inversion layer is not considered in the zero-order model framework (Conzemius and Fedorovich 2004). Despite the simplistic approach, results from studies using the zero-order jump model have shown that shear near the surface and across the inversion layer plays an important role in the growth of the boundary layer (Pino et al. 2003).

Recent large-eddy simulations (LES) (Kim et al. 2003) showed that shear can thicken the depth of the entrainment zone through the action of Kelvin-Helmholz billows. Consequently, an accurate representation of the entrainment flux must account for an inversion layer with a finite depth, which is best done using the first-order jump (FOJ) model. In this study, we develop a new entrainment parameterization using the first-order jump model for the sheared CBL, which includes the effect of surface buoyancy flux and shear at the surface and the inversion layer.

In Section 2, we derive the basic entrainment equations and then describe six LES experiments utilized for the closure of the entrainment equations. Next, the parameterizations of the entrainment flux and the inversion depth are presented. In Section 3, the developed entrainment parameterization is tested for two sheared CBLs by comparing parameterized results with results from the large-eddy simulation. Finally the conclusions are given in Section 4.

\section{Model development}

\subsection{Basic equations}

The governing equations for a convective boundary layer with shear are given by

$$
\begin{array}{r}
\frac{\partial \Theta_{\mathrm{v}}}{\partial t}=-\frac{\partial \overline{w \theta_{\mathrm{v}}}}{\partial z}-W \frac{\partial \Theta_{\mathrm{v}}}{\partial z}, \\
\frac{\partial U}{\partial t}=f\left(V-V_{\mathrm{g}}\right)-\frac{\partial \overline{u w}}{\partial z}-W \frac{\partial U}{\partial z},
\end{array}
$$




$$
\frac{\partial V}{\partial t}=-f\left(U-U_{\mathrm{g}}\right)-\frac{\partial \overline{v w}}{\partial z}-W \frac{\partial V}{\partial z}
$$

where $\Theta_{\mathrm{v}}$ is the horizontally averaged virtual potential temperature, $\overline{w \theta_{\mathrm{v}}}$ is the horizontally averaged heat flux, $W$ is the large-scale vertical velocity, $U$ and $V$ are the horizontally averaged velocity components, $\overline{u w}$ and $\overline{v w}$ are the horizontally averaged momentum fluxes, $U_{\mathrm{g}}$ and $V_{\mathrm{g}}$ are the geostrophic wind components in the $x$ and $y$ directions, respectively, and $f$ is the Coriolis parameter. Figures 1 and 2 show the idealized vertical profiles of the horizontally averaged virtual potential temperature and the wind with their corresponding vertical fluxes in a first-order jump model.

Developing the mixed-layer model starts with vertically integrating Eqs. (1)-(3) from 0 to $h_{1}$, from $h_{1}$ to $h_{2}$, and from $h_{2}$ to $h_{2}+\varepsilon$ ( $\varepsilon$ is the infinitesimal), where $h_{1}$ is the height of the heat flux minimum, and $h_{2}$ is the top of the convective boundary layer where the heat flux vanishes. The assumption is made that the mean virtual potential temperature and mean velocity components are constant over the mixed-layer depth and linearly varying between $h_{1}$ and $h_{2}$. For detailed derivations, see Appendix A. The integrations of Eq. (1) give

$$
\begin{gathered}
h_{1} \frac{\partial \Theta_{\mathrm{vm}}}{\partial t}=\overline{w \theta}_{\mathrm{vs}}-\overline{w \theta_{\mathrm{v}}} h_{1}, \\
\Delta \Theta_{\mathrm{v}} \frac{\partial h_{1}}{\partial t}=\delta \frac{\partial\left(\Theta_{\mathrm{vm}}+(1 / 2) \Delta \Theta_{\mathrm{v}}\right)}{\partial t}-\overline{w \theta}_{\mathrm{vh}_{1}}+\Delta \Theta_{\mathrm{v}} W_{h_{1}}, \\
\frac{\partial\left(\Theta_{\mathrm{vm}}+\Delta \Theta_{\mathrm{v}}\right)}{\partial t}=\gamma\left(\frac{\partial h_{1}}{\partial t}-W_{h_{1}}\right),
\end{gathered}
$$

where $\Theta_{\mathrm{vm}}$ is the mean mixed-layer virtual potential temperature, $\Delta \Theta_{\mathrm{v}}$ is the virtual potential temperature jump across $h_{1}$ and $h_{2}, \overline{w \theta_{\mathrm{vs}}}$ is the surface heat flux and $\overline{w \theta_{\mathrm{v}}} h_{1}$ is the entrainment heat flux at $z=h_{1}, \delta$ is the distance between $h_{1}$ and $h_{2}, W_{h_{1}}$ is the vertical velocity at $z=h_{1}$ and $\gamma$ is the vertical gradient of virtual potential temperature in the free atmosphere.

The integrations of Eqs. (2) and (3) give

$$
h_{1} \frac{\partial U_{\mathrm{m}}}{\partial t}=f h_{1}\left(V_{\mathrm{m}}-V_{\mathrm{g}}\right)+\overline{u w}_{\mathrm{s}}-\overline{u w}_{h_{1}},
$$

Fig. 1 Sketch of the profiles of virtual potential temperature and heat flux in a first-order jump model (modified from van Zanten et al. 1999).

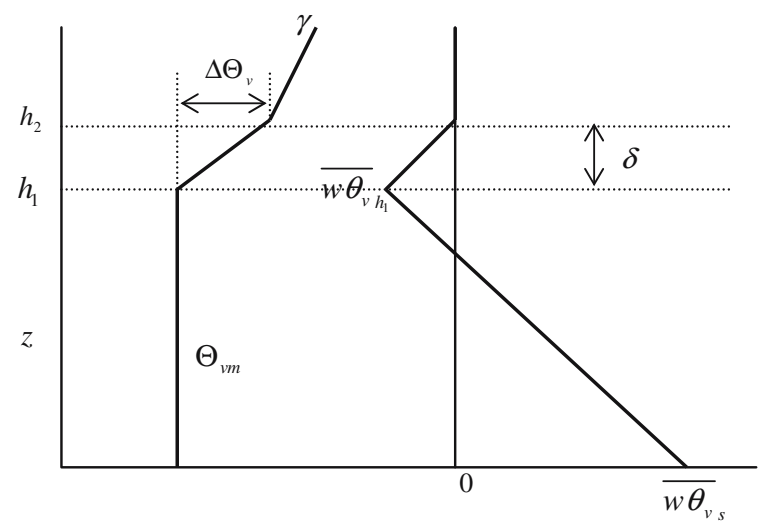



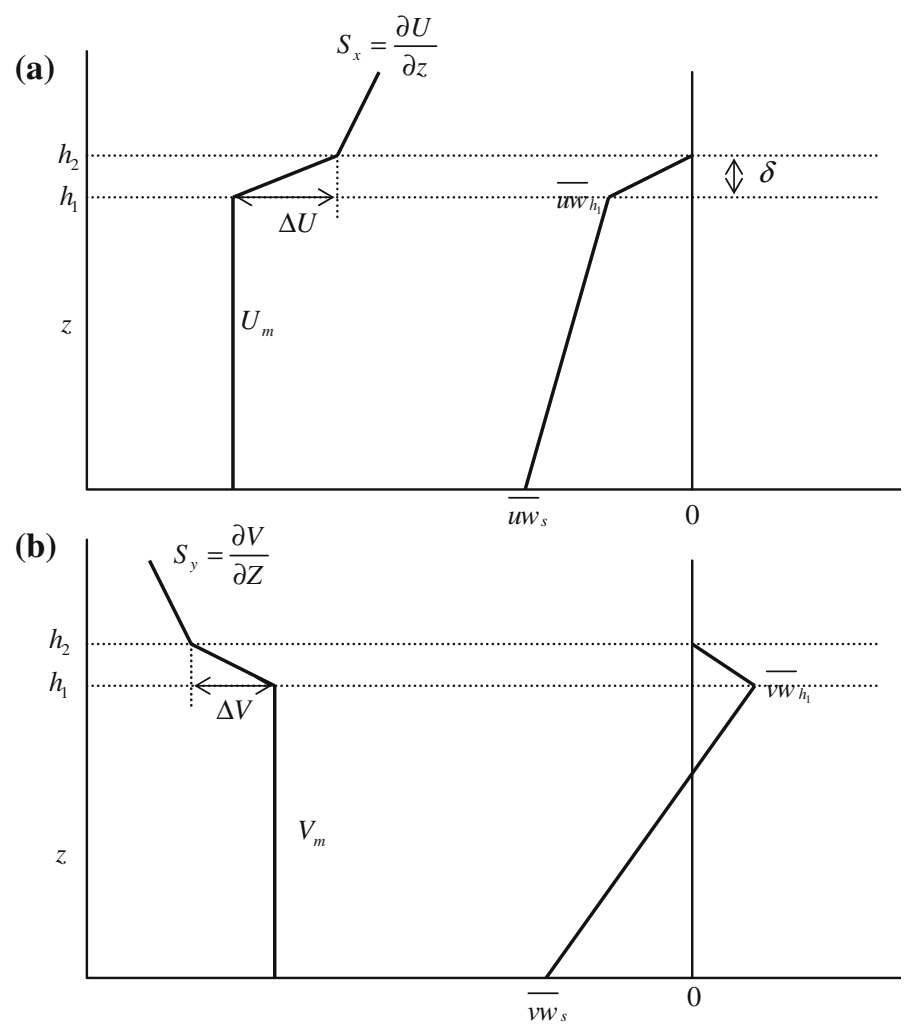

Fig. 2 Sketch of the profiles of (a) $U$ and $\overline{u w}$, (b) $V$ and $\overline{v w}$ in a first-order jump model

$$
\begin{gathered}
\Delta U \frac{\partial h_{1}}{\partial t}=\delta \frac{\partial\left(U_{\mathrm{m}}+(1 / 2) \Delta U\right)}{\partial t}-\overline{u w}_{h_{1}}+\Delta U W_{h_{1}}-f \delta\left(V_{\mathrm{m}}+\frac{\Delta V}{2}-V_{\mathrm{g}}\right), \\
\frac{\partial\left(U_{\mathrm{m}}+\Delta U\right)}{\partial t}=S_{x}\left(\frac{\partial h_{1}}{\partial t}-W_{h_{1}}\right)+f\left(V_{\mathrm{m}}+\Delta V-V_{\mathrm{g}}\right), \\
h_{1} \frac{\partial V_{\mathrm{m}}}{\partial t}=-f h_{1}\left(U_{\mathrm{m}}-U_{\mathrm{g}}\right)+{\overline{v w_{\mathrm{s}}}}-\overline{v w}_{h_{1}}, \\
\Delta V \frac{\partial h_{1}}{\partial t}=\delta \frac{\partial\left(V_{\mathrm{m}}+(1 / 2) \Delta V\right)}{\partial t}-\overline{v w}_{h_{1}}+\Delta V W_{h_{1}}+f \delta\left(U_{\mathrm{m}}+\frac{\Delta U}{2}-U_{\mathrm{g}}\right), \\
\frac{\partial\left(V_{\mathrm{m}}+\Delta V\right)}{\partial t}=S_{y}\left(\frac{\partial h_{1}}{\partial t}-W_{h_{1}}\right)-f\left(U_{\mathrm{m}}+\Delta U-U_{\mathrm{g}}\right),
\end{gathered}
$$

where $U_{\mathrm{m}}$ and $V_{\mathrm{m}}$ are mean mixed-layer velocity components, $\Delta U$ and $\Delta V$ are velocity jumps across the inversion layer, $U_{\mathrm{g}}$ and $V_{\mathrm{g}}$ are geostrophic wind components, $\overline{u w}_{\mathrm{s}}$ and $\bar{v}_{\mathrm{s}}$ are surface momentum fluxes, $\overline{u w}_{h_{1}}$ and $\overline{v w}_{h_{1}}$ are momentum fluxes at $z=h_{1}, S_{x}$ and $S_{y}$ are the free atmosphere wind shears in the $x$ and $y$ directions, respectively. 
When deriving Eqs. (4)-(12), we have assumed $W_{h_{1}}=W_{h_{1}+\frac{\delta}{2}}=W_{h_{2}}, \quad \frac{\partial \gamma}{\partial t}=\frac{\partial S_{x}}{\partial t}=$ $\frac{\partial S_{y}}{\partial t}=\frac{\partial \delta}{\partial t}=0$ (similar to van Zanten et al. 1999), and a constant divergence. Eqs. (4)-(12) have the following unknowns: $\Theta_{\mathrm{vm}}, U_{\mathrm{m}}, V_{\mathrm{m}}, \Delta \Theta_{\mathrm{v}}, \Delta U, \Delta V, \overline{w \theta}_{h_{1}}, \overline{u w}_{h_{1}}, \overline{v w}_{h_{1}}, h_{1}, \delta$. To close the equation set, $\overline{w \theta_{\mathrm{v}}} h_{1}$ and $\delta$ need to be parameterized. Merging (4) and (6) into (5) yields

$$
\left(\Delta \Theta_{\mathrm{v}}-\frac{1}{2} \gamma \delta\right)\left(\frac{\partial h_{1}}{\partial t}-W_{h_{1}}\right)=\frac{\delta}{2 h_{1}} \overline{w \theta_{\mathrm{vs}}}-\left(1+\frac{\delta}{2 h_{1}}\right) \overline{w \theta_{\mathrm{v}}} h_{1} .
$$

Equation (13) indicates that once the entrainment heat flux and the inversion layer depth are known, the entrainment rate $w_{\mathrm{e}}\left(=\frac{\partial h_{1}}{\partial t}-W_{h_{1}}\right)$ can be determined. Also, once $\overline{w \theta} h_{1}$ is known, $\partial \Theta_{\mathrm{vm}} / \partial t$ and $\partial \Delta \Theta_{\mathrm{v}} / \partial t$ can be obtained by means of Eqs. (4) and (6). Merging Eqs. (7) and (9) into (8) leads to

$$
\overline{u w}_{h_{1}}=\frac{\left[\frac{\delta}{2 h_{1}} \overline{u w}_{\mathrm{s}}-\left(\Delta U-S_{x} \frac{\delta}{2}\right)\left(\frac{\partial h_{1}}{\partial t}-W_{h_{1}}\right)\right]}{1+\frac{\delta}{2 h_{1}}} .
$$

Then, $\partial U_{\mathrm{m}} / \partial t$ and $\partial \Delta U / \partial t$ can be determined from Eqs. (7) and (9). In a similar way, from Eqs. (10), (11) and (12), the entrainment momentum flux $\overline{v w}_{h_{1}}$ is given by

$$
\overline{v w}_{h_{1}}=\frac{\left[\frac{\delta}{2 h_{1}} \overline{v w}_{\mathrm{s}}-\left(\Delta V-S_{y} \frac{\delta}{2}\right)\left(\frac{\partial h_{1}}{\partial t}-W_{h_{1}}\right)\right]}{1+\frac{\delta}{2 h_{1}}} .
$$

Then, $\partial V_{\mathrm{m}} / \partial t$ and $\partial \Delta V / \partial t$ are determined from Eqs. (10) and (12).

\subsection{Description of LES experiments}

Six CBL flows under varying geostrophic wind speeds and varying inversion strengths are simulated with the LES model to provide a basic dataset for adjusting the constants in the entrainment parameterization. The LES model used for this purpose was developed by Moeng (1984) and refined by Sullivan et al. (1994, 1996). A spectral method is used for horizontal spatial derivatives, while a centered finite difference method with a vertically staggered grid is used in the vertical direction. All prognostic quantities were integrated in time by the third-order Runge-Kutta scheme with a variable timestep (Spalart et al. 1991). A periodic boundary condition is adopted in the horizontal directions. The bottom boundary condition uses the Monin-Obukhov similarity law, and the upper boundary condition allows an upward radiation of internal gravity waves (Klemp and Durran 1983). The modified subgrid-scale model proposed by Sullivan et al. (1994) is applied.

The simulation domain is $6000 \times 6000 \times 1800 \mathrm{~m}^{3}$ in the $x, y$, and $z$ directions covered by $120 \times 120 \times 120$ grid points. The prescribed east-west component of geostrophic wind is constant with height. Depending on the numerical experiment, the geostrophic wind varies from $5 \mathrm{~m} \mathrm{~s}^{-1}$ to $15 \mathrm{~m} \mathrm{~s}^{-1}$. The north-south component of geostrophic wind is set to zero for all the experiments. The surface kinematic heat flux is fixed at $0.05 \mathrm{Km} \mathrm{s}^{-1}$. Initially, the temperature jump at the top of the boundary layer for the weak inversion cases is set to zero, whereas the temperature jump for the strong inversion cases is set to $8 \mathrm{~K}$. The initial velocity jump is set to zero for all cases. The vertical gradient of potential temperature in 
Table 1 LES simulated variables. "W" and " $S$ " in the case name represent weak and strong inversion strength, respectively

\begin{tabular}{lrrlllllll}
\hline Case & $h_{1}(\mathrm{~m})$ & $h_{2}(\mathrm{~m})$ & $\delta(\mathrm{m})$ & $\Delta \Theta_{\mathrm{V}}(\mathrm{K})$ & $\Delta U\left(\mathrm{~m} \mathrm{~s}^{-1}\right)$ & $\Delta V\left(\mathrm{~ms}^{-1}\right)$ & $u_{*}\left(\mathrm{~m} \mathrm{~s}^{-1}\right)$ & $w_{*}\left(\mathrm{~ms} \mathrm{~s}^{-1}\right)$ & $\overline{w \theta_{\mathrm{V}}} h 1 / Q_{0}$ \\
\hline $\mathrm{W} 15$ & 870 & 1065 & 195 & 1.61 & 4.55 & -2.24 & 0.67 & 1.138 & -0.28 \\
$\mathrm{~W} 10$ & 825 & 990 & 165 & 1.29 & 2.81 & -1.35 & 0.52 & 1.119 & -0.20 \\
$\mathrm{~W} 05$ & 795 & 915 & 120 & 0.94 & 0.92 & -0.45 & 0.32 & 1.105 & -0.13 \\
$\mathrm{~S} 15$ & 490 & 560 & 70 & 6.23 & 7.52 & -3.71 & 0.57 & 0.940 & -0.47 \\
$\mathrm{~S} 10$ & 480 & 540 & 60 & 6.11 & 4.45 & -2.22 & 0.45 & 0.933 & -0.31 \\
S05 & 470 & 520 & 50 & 5.52 & 1.86 & -0.96 & 0.30 & 0.926 & -0.20 \\
\hline
\end{tabular}

Numbers in the case name denote the magnitude of the geostrophic wind speed. $h_{1}$ is the height of the minimum heat flux, $h_{2}$ is the height at which heat flux disappears, $\delta$ is the inversion layer depth, $\Delta \Theta_{\mathrm{V}}$ is the virtual potential temperature jump in the inversion layer, $\Delta U$ is the east-west component of velocity jump, $\Delta V$ is the north-south component of velocity jump in the inversion layer, $u_{*}$ is the friction velocity, $w_{*}$ is the convection velocity, $\overline{w \theta}_{\mathrm{v} h 1}$ is the entrainment heat flux, and $Q_{0}$ is the surface heat flux

the free atmosphere is $0.005 \mathrm{~K} \mathrm{~m}^{-1}$. The surface roughness length is $0.16 \mathrm{~m}$, and the Coriolis parameter $f$ is fixed to a constant value of $10^{-4} \mathrm{~s}^{-1}$; the variation of $f$ with latitude can be neglected within the simulated domain.

Each simulation lasts about $12,000 \mathrm{~s}$ and data are saved for the last one hour of the simulation at approximately 100-s intervals. The parameters are averaged over 38 output samples that cover the last one hour of simulation (Table 1). As shown in Table $1, h_{2}$, the lowest height beyond which the heat flux and its vertical derivative vanishes, and $h_{1}$, the height of the maximum negative heat flux (referred to a boundary layer height), become larger with increasing wind shear. The absolute value of the entrainment heat flux relative to the surface heat flux tends to increase from 0.13 to 0.30 (from 0.2 to 0.47 ) for the weak (strong) inversion case with increasing geostrophic wind speed from $5 \mathrm{~m} \mathrm{~s}^{-1}$ to $15 \mathrm{~m} \mathrm{~s}^{-1}$.

\subsection{Parameterization of the entrainment flux}

Previous studies (Driedonks 1982; Boers et al. 1984; Gryning and Batchvarova 1990; Flamant and Pelon 1996; Betts and Barr 1996; Flamant et al. 1997; Pino et al. 2003) have used the turbulent kinetic energy (TKE) equation to parameterize the entrainment heat flux. This parameterization of the entrainment heat flux can be developed by either using a local TKE budget or using an integrated TKE budget. First, we compare the parameterization of Tennekes and Driedonks (1981) with the LES results described in Section 2.2. This popular scheme is one example of the local TKE budget approach.

\subsubsection{An example of the parameterization using local turbulent kinetic energy budget}

The parameterization of the entrainment heat flux using the TKE budget at the mixed-layer height, $h_{1}$, in the framework of a zero-order jump model (Tennekes and Driedonks 1981) is given as

$$
-\frac{g}{\Theta_{\mathrm{v} 0}} \overline{w \theta_{\mathrm{v}}} h_{1}=-C_{\mathrm{T}} \frac{w_{\mathrm{m}}^{2}}{h_{1}} \frac{d h_{1}}{d t}+C_{\mathrm{F}} \frac{w_{\mathrm{m}}^{3}}{h_{1}}-C_{\mathrm{D}} w_{\mathrm{m}}^{2} N+C_{\mathrm{M}}\left(\Delta U^{2}+\Delta V^{2}\right) \frac{1}{h_{1}} \frac{d h_{1}}{d t},
$$

where $w_{\mathrm{m}}^{2}=w_{*}^{2}+4 u_{*}^{2}$ and $C_{\mathrm{F}}=0.6, C_{\mathrm{T}}=4.3, C_{\mathrm{D}}=0.03$, and $C_{\mathrm{M}}=1$. The left-hand side of Eq. (16) is the buoyancy term of the TKE equation. The first term on the right-hand side represents the rate of change of TKE, the second term the sum of transport of TKE 
Table 2 Entrainment closure ratios (absolute value of entrainment heat flux normalized by surface heat flux) derived by LES data and by Eq. (16)

\begin{tabular}{llll}
\hline Case & LES & EQ 16 & Difference (\%) \\
\hline W15 & 0.28 & 1.36 & 387 \\
W10 & 0.20 & 0.87 & 337 \\
W05 & 0.13 & 0.47 & 261 \\
S15 & 0.47 & 1.90 & 305 \\
S10 & 0.31 & 1.28 & 313 \\
S05 & 0.20 & 0.78 & 288 \\
\hline
\end{tabular}

and pressure transport, the third term the dissipation, and the fourth term the TKE generated by velocity jumps at the top of the mixed layer. Table 2 shows the comparison between the parameterized entrainment heat fluxes (normalized by the surface heat flux) determined by Eq. (16) and the entrainment heat fluxes derived from the LES data. As shown, the parameterized entrainment heat fluxes calculated by Eq. (16) are three to four times larger than the LES results. This is mainly due to the overestimation of wind shear production, the transport of TKE, and the pressure transport terms. The parameterization discussed above is one example of local TKE budget approach. It does not imply that every local TKE budget approach produces incorrect results. Pino et al. (2003) showed that the entrainment heat fluxes from the modified version of Eq. (16) in their zero-order jump approach agree well with LES results. However, the local TKE approach does have difficulty in determining an accurate and representative TKE budget at a single level, $z=h_{1}$. For this reason, we develop a parameterization based on the integrated TKE budget.

\subsubsection{New parameterization using integrated turbulent kinetic energy budget}

The vertically integrated TKE budget from the surface to the top of the CBL $\left(h_{2}\right)$ reads

$$
\begin{aligned}
\int_{0}^{h_{2}} \frac{d E}{d t} d z= & \int_{0}^{h_{2}} \frac{g}{\Theta_{\mathrm{v} 0}} \overline{w \theta_{\mathrm{v}}} d z-\int_{0}^{h_{2}}\left[\overline{u w} \frac{\partial U}{\partial Z}+\overline{v w} \frac{\partial V}{\partial Z}\right] d z \\
& -\int_{0}^{h_{2}}\left[\frac{\partial}{\partial z}(\overline{w p}+\overline{w E})\right] d z+\int_{0}^{h_{2}} \varepsilon d z .
\end{aligned}
$$

The left-hand side of Eq. (17) is the rate of change of TKE (E) storage in the CBL. Each term on the right-hand side of Eq. (17), in order from left to right, is the total buoyancy production/consumption of TKE, the total TKE production by wind shear, the vertical redistribution, and the viscous dissipation. As in Driedonks (1982) and Randall (1984), the left-hand side of Eq. (17) is small compared to other terms except for the early stage of the CBL growth. The LES data we use for the determination of the empirical coefficients are in quasi-steady state when the time change of TKE is negligible. Thus, in this study, we ignore the temporal change of the TKE term. This assumption can be a possible source of error at the early stage of CBL development. The third term on the right-hand side of Eq. (17) is set to zero as found in our LES results (see also Moeng and Sullivan 1994). There may be an energy flux at the top of the CBL produced by propagating internal gravity waves. Our knowledge about this energy flux generated by internal gravity waves is not sufficient to parameterize it because measurement of pressure fluctuations in the atmosphere is very difficult (Stull 1988). Thus, 
in developing our parameterization, we set the energy fluxes at the surface and the top of the CBL to zero.

By assuming a linear variation of fluxes through the mixed layer, the integrated buoyancy term in Eq. (17) becomes

$$
\int_{0}^{h_{2}} \frac{g}{\Theta_{\mathrm{v} 0}} \overline{w \theta_{\mathrm{v}}} d z=\frac{1}{2} \frac{g}{\Theta_{\mathrm{v} 0}} \overline{w \theta_{\mathrm{vs}}} h_{1}+\frac{1}{2} \frac{g}{\Theta_{\mathrm{v} 0}} \overline{w \theta_{\mathrm{v}}} h_{1}\left(h_{1}+\delta\right),
$$

and the integrated shear production term is

$$
-\int_{0}^{h_{2}}\left[\overline{u w} \frac{\partial U}{\partial Z}+\overline{v w} \frac{\partial V}{\partial Z}\right] d z=C_{\mathrm{D}}^{-1 / 2} u_{*}^{3}+\frac{1}{2} \frac{\delta u_{*}^{2}\left(\frac{U_{\mathrm{m}}}{M} \Delta U+\frac{V_{\mathrm{m}}}{M} \Delta V\right)+2 h_{1}(\Delta \tilde{U})^{2} w_{\mathrm{e}}}{2 h_{1}+\delta},
$$

where $C_{\mathrm{D}}$ is the drag coefficient, $M=\sqrt{U_{\mathrm{m}}^{2}+V_{\mathrm{m}}^{2}}$ and $(\Delta \tilde{U})^{2}=(\Delta U)^{2}+(\Delta V)^{2}$. See Appendix B for the detailed derivation of Eq. (19). Strictly speaking, as in the inversion layer, if temperature is a linear function of $z$, its flux is a quadratic function of $z$. To make the derivations as simple as possible, we assumed the fluxes as linear functions of $z$. The entrainment flux determined from the linear flux profile in the inversion layer is overestimated relative to the one calculated by considering the curvature of the flux profile. We ignore this error in the present study. However, the use of the corrected entrainment fluxes for the first-order jump modelling framework or the development of parameterization using the realistic profiles is an important topic for further investigation. Finally, the integration of the dissipation term results in

$$
\int_{0}^{h_{2}} \varepsilon d z=\alpha_{1} \frac{1}{2} \frac{g}{\Theta_{\mathrm{v} 0}} \overline{w \theta_{\mathrm{vs}}} h_{1}+\alpha_{2} C_{\mathrm{D}}^{-1 / 2} u_{*}^{3}+\alpha_{3} \frac{1}{2} \frac{\delta u_{*}^{2}\left(\frac{U_{\mathrm{m}}}{M} \Delta U+\frac{V_{\mathrm{m}}}{M} \Delta V\right)+2 h_{1}(\Delta \tilde{U})^{2} w_{\mathrm{e}}}{2 h_{1}+\delta},
$$

where $\alpha_{1}, \alpha_{2}$, and $\alpha_{3}$ are constants. In deriving Eq. (20), the total dissipation of TKE in the whole boundary layer is assumed to be proportional to the production of TKE. We followed the approach of Flamant et al. (1999).

The integrated total TKE budget in the parameterized form is given by

$$
\begin{aligned}
-\frac{g}{\Theta_{\mathrm{v} 0}} \overline{w \theta_{\mathrm{v}}} h_{1}\left(h_{1}+\delta\right)= & A_{1} w_{*}^{3}+A_{2} u_{*}^{3} \\
& +A_{3}\left[\frac{\delta u_{*}^{2}}{4 h_{1}+2 \delta}\left(\frac{U_{\mathrm{m}}}{M} \Delta U+\frac{V_{\mathrm{m}}}{M} \Delta V\right)+\frac{h_{1}(\Delta \tilde{U})^{2} w_{\mathrm{e}}}{2 h_{1}+\delta}\right],
\end{aligned}
$$

where $A_{1}=1-\alpha_{1}, A_{2}=2 C_{\mathrm{D}}^{-1 / 2}\left(1-\alpha_{2}\right), A_{3}=2\left(1-\alpha_{3}\right), w_{*}^{3}=\frac{g}{\Theta_{\mathrm{v} 0}} \overline{w \theta_{\mathrm{vs}}} h_{1}$. In the present study, we treat $A_{2}$ as a constant. This assumption may not be correct since it does not incorporate the effect of various surface roughness lengths. However, for simplification, we set it as a constant such as is done in Flamant et al. (1999). 


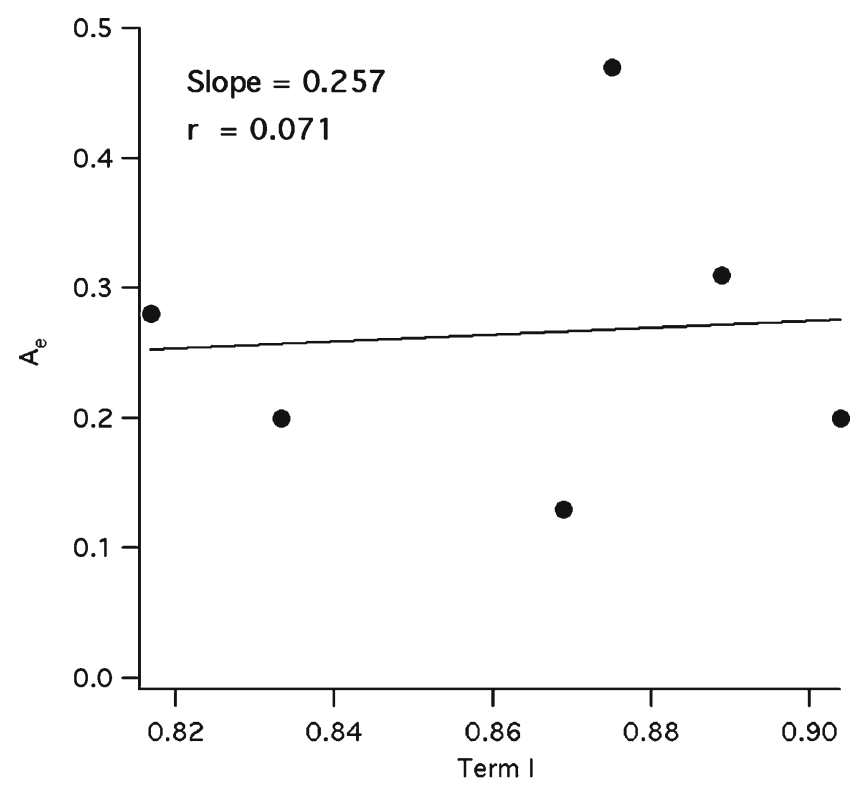

Fig. 3 The relationship between term I and $A_{\mathrm{e}}$ in Eq. (22). The circle represents data from the LES model. The line is the least squares fit to the LES data

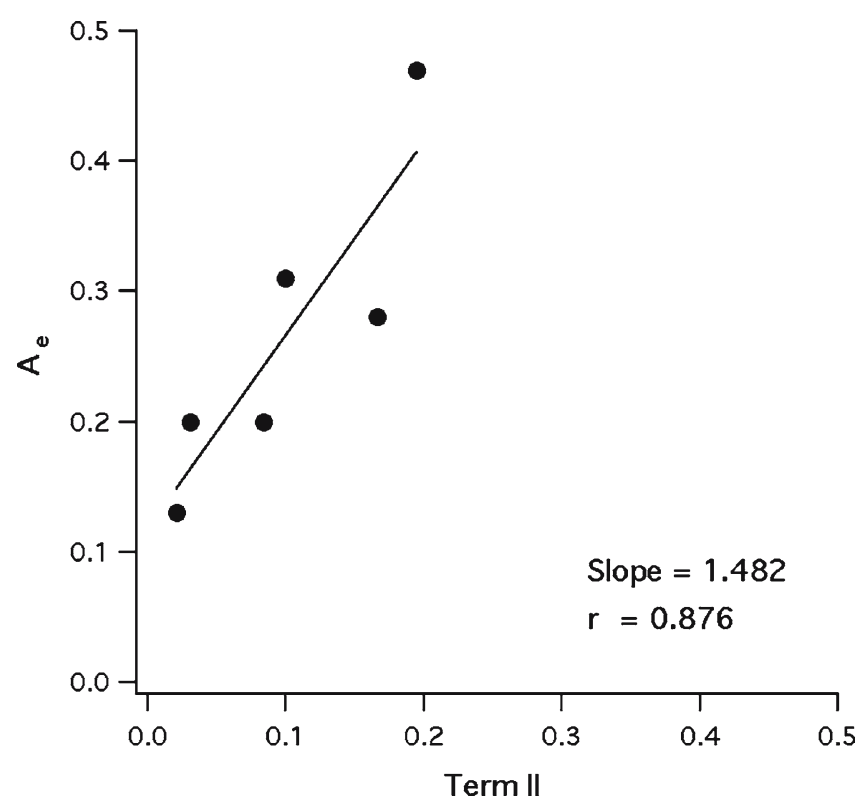

Fig. 4 The same as in Fig. 3 except for term II 
The entrainment ratio is

$$
\begin{aligned}
A_{\mathrm{e}} & =-\frac{\overline{w \theta_{\mathrm{v}}} h_{1}}{\overline{w \theta_{\mathrm{vs}}}} \\
& =A_{1} \frac{1}{1+\delta / h_{1}}+A_{2} \frac{u_{*}^{3}}{w_{*}^{\prime 3}}+A_{3} \frac{1}{w_{*}^{\prime 3}}\left[\frac{\delta u_{*}^{2}}{4 h_{1}+2 \delta}\left(\frac{U_{\mathrm{m}}}{M} \Delta U+\frac{V_{\mathrm{m}}}{M} \Delta V\right)+\frac{h_{1}(\Delta \tilde{U})^{2} w_{\mathrm{e}}}{2 h_{1}+\delta}\right],
\end{aligned}
$$

where, $w_{*}^{\prime 3}=\frac{g}{\Theta_{\mathrm{v} 0}} \overline{w \theta_{\mathrm{vs}}}\left(h_{1}+\delta\right)$. From Eq. (13), the entrainment velocity $w_{\mathrm{e}}$ is given by

$$
w_{\mathrm{e}}=\frac{\frac{\delta}{2 h_{1}} \overline{w \theta}_{\mathrm{s}}-\left(1+\frac{\delta}{2 h_{1}}\right) \overline{w \theta}_{h_{1}}}{\left(\Delta \Theta_{\mathrm{v}}-\frac{1}{2} \gamma \delta\right)} .
$$

Replacing $w_{\mathrm{e}}$ in Eq. (22) by Eq. (23) yields

$$
\begin{aligned}
& A_{\mathrm{e}}=-\frac{\overline{w \theta_{\mathrm{v}}} h_{1}}{\overline{w \theta_{\mathrm{vs}}}} \\
& =\frac{A_{1} \frac{1}{1+\delta / h_{1}}+A_{2} \frac{u_{*}^{3}}{w_{*}^{\prime 3}}+A_{3} \frac{1}{w_{*}^{\prime 3}} \frac{\delta}{\left(4 h_{1}+2 \delta\right)}\left[u_{*}^{2}\left(\frac{U_{\mathrm{m}}}{M} \Delta U+\frac{V_{\mathrm{m}}}{M} \Delta V\right)+\frac{\overline{w \theta_{\mathrm{vs}}}}{\left(\Delta \Theta_{\mathrm{v}}-\frac{1}{2} \gamma \delta\right)}(\Delta \tilde{U})^{2}\right]}{\left[1-\frac{(\Delta \tilde{U})^{2}}{2 \frac{g}{\Theta_{\mathrm{v} 0}}\left(\Delta \Theta_{\mathrm{v}}-\frac{1}{2} \gamma \delta\right)\left(h_{1}+\delta\right)} A_{3}\right]} .
\end{aligned}
$$

If wind shear is neglected, then Eq. (24) reduces to

$$
A_{\mathrm{e}}=A_{1} \frac{1}{1+\delta / h_{1}} .
$$

In Eq. (25), $\delta / h_{1}$ is known to be inversely proportional to the convective Richardson number, $R i_{*}=\frac{\left(g / \Theta_{0}\right) \Delta \Theta_{\mathrm{v}} h_{1}}{w_{*}^{2}}$ (Driedonks 1984) or the stability of the inversion layer. Depending on the inversion strength $A_{\mathrm{e}}$ becomes close to $A_{1}$ (strong inversion) or $A_{\mathrm{e}}$ becomes less than $A_{1}$ (weak inversion). For instance, $A_{\mathrm{e}}$ is 0.2 for the $\mathrm{S} 05$ experiment, while $A_{\mathrm{e}}$ is 0.13 for W05.

We obtain coefficients $A_{1}$ to $A_{3}$ by applying multiple linear regression analysis, to give $A_{1}=0.35, A_{2}=1.36$, and $A_{3}=0.44 . A_{1}=0.35$ is too big relative to the classical closure value of 0.2 for a CBL with negligible shear. This discrepancy may reflect the limitation of our dissipation parameterization or assumption in the first-order jump model. Figures 3, 4 and 5 illustrate whether the multiplication factors of $A_{1}, A_{2}$, and $A_{3}$ in Eq. (22) can be assumed to be linearly proportional to the normalized entrainment heat flux. The multiplication factors of $A_{1}, A_{2}$ and $A_{3}$ in Eq. (22) are given by

$$
\begin{gathered}
\text { Term } \mathrm{I}=1 /\left(1+\delta / h_{1}\right), \\
\text { Term II }=u_{*}^{3} / w_{*}^{\prime 3}, \\
\text { Term III }=\left(1 / w_{*}^{\prime 3}\right)\left[\delta /\left(4 h_{1}+2 \delta\right) u_{*}^{2}\left(\frac{U_{\mathrm{m}}}{M} \Delta U+\frac{V_{\mathrm{m}}}{M} \Delta V\right)+h_{1}(\Delta \tilde{U})^{2} w_{\mathrm{e}} /\left(2 h_{1}+\delta\right)\right] .
\end{gathered}
$$




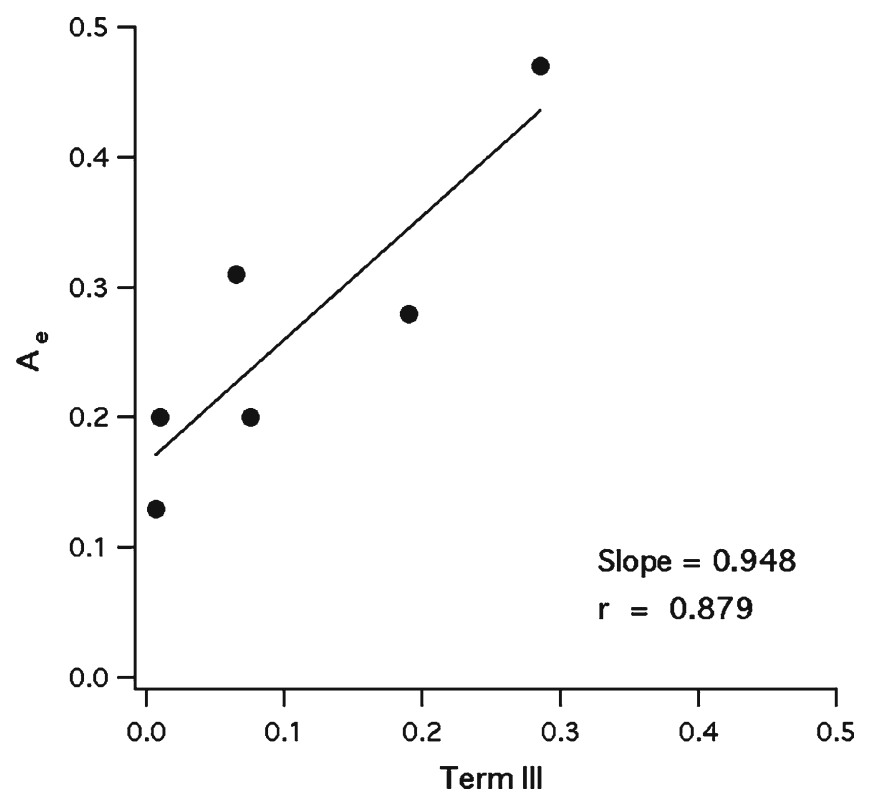

Fig. 5 The same as in Fig. 3 except for term III

Table 3 Entrainment closure ratios (absolute value of entrainment heat flux normalized by surface heat flux) derived by LES data and by Eqs. (24) and (26)

\begin{tabular}{lccccc}
\hline Case & LES & EQ24 & EQ26 & \multicolumn{2}{c}{ Error (\%) } \\
\cline { 5 - 6 } & & & & EQ24 & EQ26 \\
\hline W15 & 0.28 & 0.35 & 0.35 & 25 & 25 \\
W10 & 0.20 & 0.25 & 0.23 & 25 & 15 \\
W05 & 0.13 & 0.19 & 0.17 & 46 & 31 \\
S15 & 0.47 & 0.43 & 0.44 & -9 & -6 \\
S10 & 0.31 & 0.27 & 0.26 & -13 & -16 \\
S05 & 0.20 & 0.20 & 0.20 & 0 & 0 \\
\hline
\end{tabular}

Figure 3 shows that the relationship between Term I and $A_{\mathrm{e}}$ is not clear. However, from Figs. 4 and $5, A_{\mathrm{e}}$ is approximately linearly proportional to Term II and Term III. Uncertainties in the estimation of $h_{2}$ may be one possible reason for the poor correlation between Term I and $A_{\mathrm{e}}$. Since the LES heat flux profile above $h_{1}$ does not converge to zero quickly and sometimes shows positive and negative fluctuations near $h_{2}$, estimation of $\delta$ is not straightforward. To develop the parameterization, hereafter, we set $A_{1}=0.2$, which is the value for the strong inversion case (S05) and also the classical closure value for CBL with negligible shear. Using $A_{1}=0.2, A_{2}$ and $A_{3}$ are determined by the multiple linear regression of LES. Thus, by using multiple regression to determine $A_{2}$ and $A_{3}$, we find $A_{2}=0.6$ and $A_{3}=0.474$. The parameterized entrainment flux ratios in Eq. (24) with $A_{1}=0.2, A_{2}=0.6, A_{3}=0.474$ are compared with ones from LES data in Table 3 . The parameterized entrainment flux ratio is in good agreement with those calculated from LES data. The difference ranges from $-13 \%$ to $46 \%$.

The other form of entrainment closure ratio is proposed because Eq. (24) includes the signs of $\Delta U$ and $\Delta V$, and effects of direction of elevated wind shear on the entrainment rate are still being debated (e.g., Fedorovich et al. 2001). 


$$
\begin{aligned}
A_{\mathrm{e}} & =-\frac{\overline{w \theta_{\mathrm{v}}} h_{1}}{\overline{w \theta_{\mathrm{vs}}}} \\
& =\frac{A_{1} \frac{1}{1+\delta / h_{1}}+A_{2} \frac{u_{*}^{3}}{w_{*}^{\prime 3}}+A_{3} \frac{1}{w_{*}^{\prime 3}} \frac{\delta}{\left(4 h_{1}+2 \delta\right)}\left[u_{*}^{2}(\Delta \hat{U})+\frac{\overline{w \theta_{\mathrm{v} s}}}{\left(\Delta \Theta_{\mathrm{v}}-\frac{1}{2} \gamma \delta\right)}(\Delta \hat{U})^{2}\right]}{\left[1-\frac{(\Delta \hat{U})^{2}}{2 \frac{g}{\Theta_{\mathrm{v} 0}}\left(\Delta \Theta_{\mathrm{v}}-\frac{1}{2} \gamma \delta\right)\left(h_{1}+\delta\right)} A_{3}\right]},
\end{aligned}
$$

where $\Delta \hat{U}=0.5(|\Delta U|+|\Delta V|), A_{1}=0.2, A_{2}=0.26$, and $A_{3}=1.44$. The parameterized entrainment flux ratios in Eq. (26) are also compared with ones from LES data in Table 3. The parameterized entrainment flux ratio from Eq. (26) is also in good agreement with those calculated from LES data. The difference ranges from $-16 \%$ to $31 \%$. Equation (26) is introduced because it is used in Pino et al. (2006), a mixed-layer model comparison study.

\subsection{Parameterization of the inversion layer depth}

To solve the basic entrainment equations in a first-order jump model, the inversion layer depth, $\delta\left(=h_{2}-h_{1}\right)$ must be estimated. A parcel method is applied to diagnose $\delta$. If the wind shear across the inversion layer is negligible, the parcel method yields

$$
\frac{\delta}{h_{1}}=\frac{w_{\mathrm{c}}^{2}}{\left(g / \Theta_{\mathrm{v} 0}\right) \Delta \Theta_{\mathrm{v}} h_{1}}
$$

where $w_{\mathrm{c}}$ is the characteristic velocity associated with the impinging of thermals at the base of the inversion layer. We assume that $w_{\mathrm{c}}$ depends on the friction velocity and the convection velocity, $w_{\mathrm{c}}^{2} \sim f\left(w_{*}^{2}, u_{*}^{2}\right)$. If a large wind shear exists at the inversion layer, $\delta$ is enhanced due to greater TKE compared to the situation without wind shear in the inversion layer. The effect of the increased wind shear in the inversion layer on $\delta$ is the same as the effect of the decreased temperature jump in the inversion layer on $\delta$. A modified temperature jump that incorporates the impact of wind shear across the inversion layer can be defined as

$$
\Delta \Theta_{\mathrm{v} \bmod }=\Delta \Theta_{\mathrm{v}}\left(1-R i_{\mathrm{IL}}^{-1}\right),
$$

where $R i_{\mathrm{IL}}=\frac{\left(g / \Theta_{0}\right) \Delta \Theta_{\mathrm{v}} \delta}{w_{\mathrm{IL}}^{2}}$ is a newly defined Richardson number in the inversion layer and $w_{\mathrm{IL}}^{2} \sim f\left(\Delta U^{2}, \Delta V^{2}\right)$. When wind shear across the inversion layer is considered, Eq. (27) reads

$$
\frac{\delta}{h_{1}}=\frac{w_{\mathrm{c}}^{2}}{\left(g / \Theta_{\mathrm{v} 0}\right) \Delta \Theta_{\mathrm{vmod}} h_{1}} .
$$

By rearranging Eq. (29) with Eq. (28), the inversion layer depth is

$$
\frac{\delta}{h_{1}}=a R i^{-1}+b
$$

where $R i=\frac{\left(g / \Theta_{\mathrm{v} 0}\right) \Delta \Theta_{\mathrm{v}} h_{1}}{w_{\delta}^{2}}, w_{\delta}^{2}=w_{*}^{2}+c u_{*}^{2}+d\left(\Delta U^{2}+\Delta V^{2}\right)$, and $a, b, c$, and $d$ are constants. The constants $a, b, c$, and $d$ are found by a least squares fit to the LES datasets resulting in $a=0.075, b=0.0925, c=180.21$, and $d=0.15$ with a non-zero $y$-intercept $\left(r^{2}=0.999\right)$. If we apply multiple linear regression with the $y$-intercept $=0, a=2.68, b=0$, $c=2.99, d=0.03\left(r^{2}=0.958\right)$. We also include the inversion layer parameterization with coefficients $a=1.12$ and $b=0.08$ with an ad-hoc choice of $c=4$ and $d=0.1$ because 
Table 4 Parameterization formulae for the inversion layer depth

\begin{tabular}{ll}
\hline IL1 & $\frac{\delta}{h_{1}}=0.075 R i^{-1}+0.0925, \quad w_{\delta}^{2}=w_{*}^{2}+180.21 u_{*}^{2}+0.15\left(\Delta U^{2}+\Delta V^{2}\right)$ \\
IL2 & $\frac{\delta}{h_{1}}=2.68 R i^{-1}, \quad w_{\delta}^{2}=w_{*}^{2}+2.99 u_{*}^{2}+0.03\left(\Delta U^{2}+\Delta V^{2}\right)$ \\
IL3 & $\frac{\delta}{h_{1}}=1.12 R i^{-1}+0.08, \quad w_{\delta}^{2}=w_{*}^{2}+4 u_{*}^{2}+0.1\left(\Delta U^{2}+\Delta V^{2}\right)$ \\
\hline
\end{tabular}

Table 5 Initial data for the first-order jump model

\begin{tabular}{lllllllll}
\hline Case & $h_{1}(\mathrm{~m})$ & $\delta(\mathrm{m})$ & $\Theta_{\mathrm{vm}}(\mathrm{K})$ & $\Delta \Theta_{\mathrm{V}}(\mathrm{K})$ & $U_{\mathrm{m}}\left(\mathrm{m} \mathrm{s}^{-1}\right)$ & $\Delta U\left(\mathrm{~ms}^{-1}\right)$ & $V_{\mathrm{m}}\left(\mathrm{m} \mathrm{s}^{-1}\right)$ & $\Delta V\left(\mathrm{~m} \mathrm{~s}^{-1}\right)$ \\
\hline $\mathrm{W} 20$ & 750 & 250 & 301.75 & 1.20 & 16.50 & 3.50 & 0.83 & -0.83 \\
$\mathrm{~S} 20$ & 704 & 190 & 303.16 & 2.16 & 14.93 & 5.07 & 1.85 & -1.85 \\
\hline
\end{tabular}

Pino et al. (2006) used Eq. (26) and this inversion layer depth formula in their comparison of mixed-layer models. For summary, the inversion layer formulae are listed in Table 4.

\section{Evaluation of the parameterization}

In order to evaluate the first-order jump model developed in Section 2, the model is tested for two LES generated CBLs with stronger wind shear and more intense surface heating conditions. The time evolution of the boundary-layer height $\left(h_{1}\right)$, entrainment closure ratio $\left(A_{\mathrm{e}}\right), \Theta_{\mathrm{vm}}, \Delta \Theta_{\mathrm{v}}, U_{\mathrm{m}}, \Delta U, V_{\mathrm{m}}$ and $\Delta V$ from our first-order jump model are compared with those derived from new LES data. The LES model used in this section is described in Cuijpers and Holtslag (1998), which has previously been used in the shear-driven CBL by Pino et al. (2003). The two LES models give similar results for predicting entrainment into the sheared convective boundary layer (Fedorovich et al. 2004). Thus, the evaluation of the first-order jump model parameterization should give results comparable to those of the LES described earlier. The simulation domain is $10000 \times 10000 \times 2000 \mathrm{~m}^{3}$ in the $x, y$, and $z$ directions covered by $256 \times 256 \times 64$ grid points. The geostrophic wind speed and initial wind profiles in the LES are $U_{\mathrm{g}}=20 \mathrm{~m} \mathrm{~s}^{-1}, V_{\mathrm{g}}=0 \mathrm{~m} \mathrm{~s}^{-1}$ constant with height and the surface kinematic heat flux is $0.1 \mathrm{Km} \mathrm{s}^{-1}$. Two cases characterized by different inversion strengths were simulated. The initial slope of the potential temperature vertical profile has a constant value in the whole domain of $0.003 \mathrm{~K} \mathrm{~m}^{-1}$ and $0.006 \mathrm{~K} \mathrm{~m}^{-1}$ for the weak and strong inversion cases, respectively. A more detailed description of the LES simulations is presented by Fedorovich et al. (2004). The initial data for the first-order jump model are summarized in Table 4.

The time evolutions of $h_{1}, \delta, A_{e}, \Theta_{\mathrm{vm}}, \Delta \Theta_{\mathrm{v}}, U_{\mathrm{m}}, \Delta U, V_{\mathrm{m}}$ and $\Delta V$ are shown in Figs. 6 and 7 for both the first-order jump model and the LES model for the weak and strong inversion cases, respectively. Three versions of the first-order jump model are tested. The first is the combination of Eq. (24) and IL1 in Table 4 (hereafter FOJ1). The second is the same as the first version except for the inversion layer depth parameterization, IL2 in Table 4 (hereafter FOJ2). The last is the version using Eq. (26) and IL3 in Table 4, which is tested in Pino et al. (2006) (hereafter FOJ3). Overall, three versions of the first-order jump model perform similarly and show good agreement with LES data for both stability regimes.

For the weak inversion case, the mixed-layer height prediction from FOJ2 shows the best agreement with LES data (Fig. 6a); $A_{\mathrm{e}}$ from FOJ2 is also closest to the LES data (Fig. 6b). However, it should be mentioned that $\delta$ from FOJ2 is overestimated compared to LES (Fig. 6a). The $\delta$ values from FOJ1 and FOJ3 is similar and close to those from LES. The $A_{\mathrm{e}}$ values from FOJ1 and FOJ3 are also in reasonable agreement with LES. However, the mixed-layer height predicted by FOJ1 and FOJ3 are slightly underestimated. The $\Theta_{\mathrm{vm}}$ 

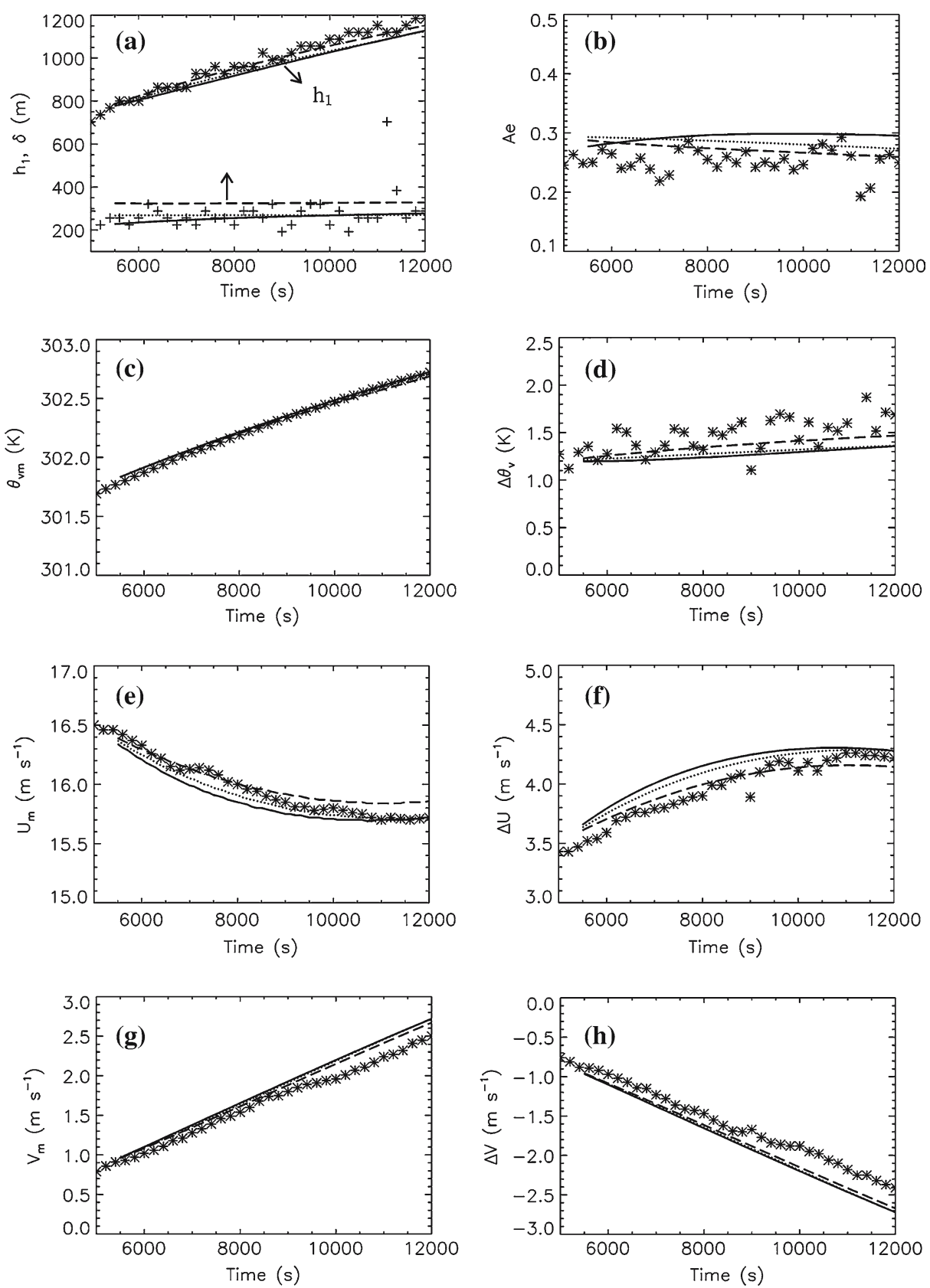

Fig. 6 Comparison of mixed-layer model results (lines) with LES (symbols) for the weak inversion case: (a) boundary-layer height $h_{1}$ and inversion layer depth $\delta$, (b) entrainment closure ratio $A_{\mathrm{e}}$, (c) $\Theta_{\mathrm{vm}},(\mathrm{d}) \Delta \Theta$, (e) $U_{\mathrm{m}}$, (f) $\Delta U$, (g) $V_{\mathrm{m}}$, and (h) $\Delta \mathrm{V}$. Dotted lines denote the model output with Eq. (24) and IL1 in Table 4 (FOJ1), dashed lines show the results with Eq. (24) and IL2 (FOJ2), and solid lines indicate the results with Eq. (26) and IL3 (FOJ3), which is the same as in Pino et al. (2006) 

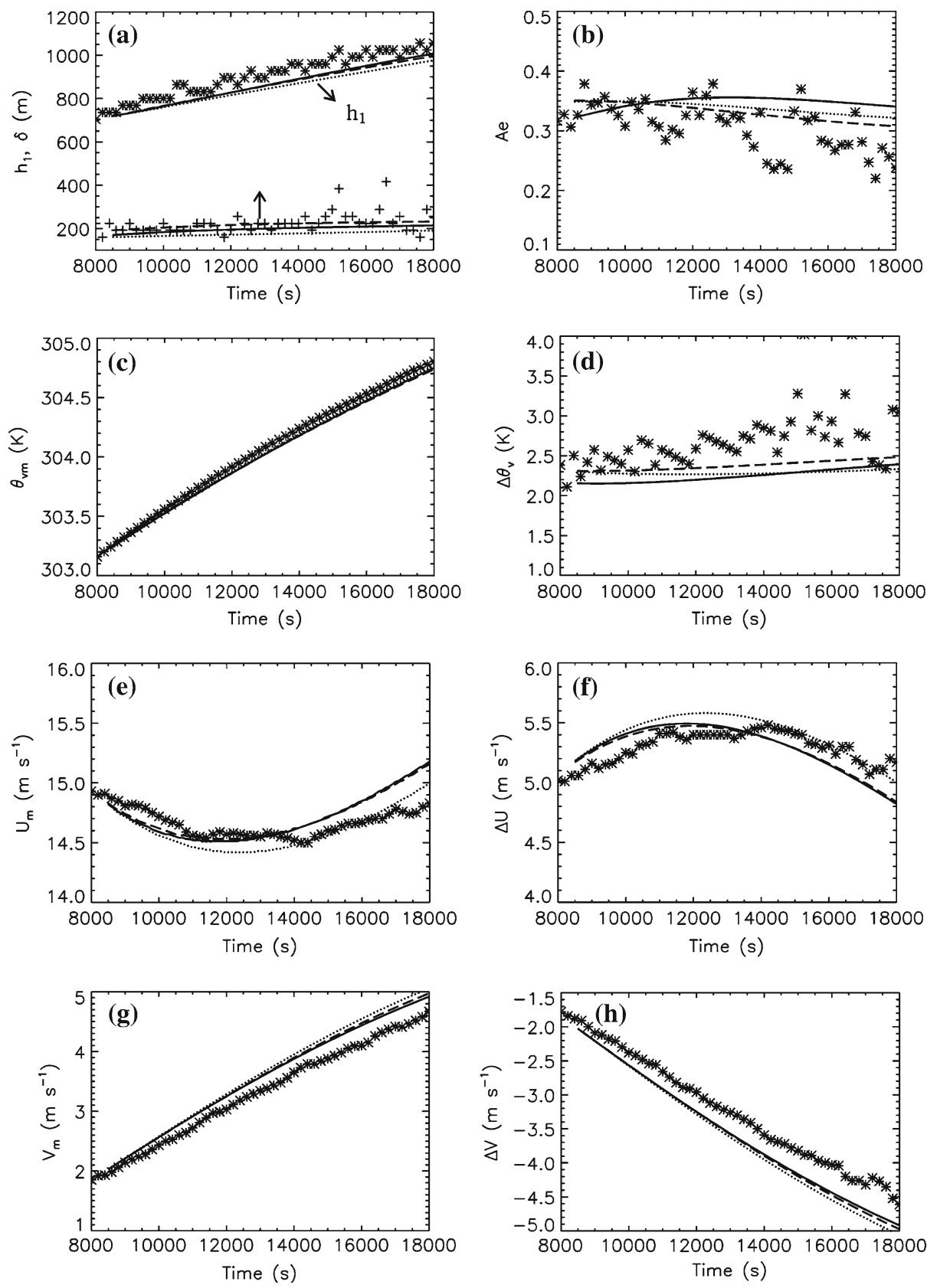

Fig. 7 The same as in Fig. 6 except for the strong inversion case

values from all of the models are in quite good agreement with LES data. The $\Delta \Theta_{\mathrm{v}}$ values from FOJ2 are closer to LES data than those from FOJ1 and FOJ3 (Fig. 6d). Compared to the $\Theta_{\mathrm{vm}}$ values, the $\Delta \Theta_{\mathrm{v}}$ values are less similar to LES data. Greater $\Delta \Theta_{\mathrm{v}}$ fluctuations in LES are associated with greater $h_{2}$ fluctuations in LES. Since a strong temperature gradient exists in the inversion layer and free atmosphere, the $\Delta \Theta_{\mathrm{v}}$ value is quite sensitive to the small 
fluctuation in $h_{2}$. The model-simulated $U_{\mathrm{m}}, \Delta U, V_{\mathrm{m}}$ and $\Delta V$ values are also in reasonable agreement with LES data (Fig. 6e-h). It is interesting that FOJ1 and FOJ3, which diagnose the inversion layer depth similarly, predict $\Theta_{\mathrm{vm}}, \Delta \Theta_{\mathrm{v}}, U_{\mathrm{m}}, \Delta U, V_{\mathrm{m}}$ and $\Delta V$ quite closely. Now a question can be raised: why does the model with $A_{\mathrm{e}}$ closest to LES and overestimated inversion layer depth predict the mixed-layer height best? One possible reason is the underestimation of the inversion layer depth from LES. Pino et al. (2006) indicated that the inversion layer depth from LES used in both this study and their study may be slightly underestimated. Thus, it implies that the inversion layer depths from FOJ1 and FOJ3 may be underestimated. Following Eq. (23), underestimation of the inversion layer depth leads to underestimation of the entrainment rate. Uncertainties in the inversion layer depth derived from LES should be kept in mind. The other possible reason is associated with the definitions of entrainment heat flux in the first-order jump model and in LES data. The entrainment heat flux, $\overline{w \theta_{\mathrm{v}}} h_{1}$, which in the first-order jump model is determined by a linear extension of the heat flux profile from the surface to $h_{1}$, should be greater than $\overline{w \theta_{\mathrm{v}}} h_{1}$ calculated by the LES. Therefore, the slight overestimation of $A_{\mathrm{e}}$ may be required to predict mixed-layer height better due to the curved heat flux profile in the LES. If this is true, the overestimated inversion layer depth from FOJ2 compensates the error from the underestimation of $A_{\mathrm{e}}$, which, in turn, predicts the mixed-layer height correctly. This suggests that a way to decrease the discrepancies of the entrainment heat flux between the first-order jump model and LES needs to be found.

For strong inversion cases, the mixed-layer heights predicted by three first-order jump models are slightly underestimated compared to those from LES. FOJ2 and FOJ3 simulated the mixed-layer height better than FOJ1 (Fig. 7a). The $A_{\mathrm{e}}$ values from three models are in good agreement with those from LES; the $A_{\mathrm{e}}$ values from FOJ1 lie between FOJ2 and FOJ3 (Fig. 7b). It should be mentioned that the inversion layer depth from FOJ1 is much underestimated, which leads to an underestimation of the entrainment rate following Eq. (23). The inversion layer depths from FOJ2 and FOJ3 are similar to those from LES. Thus, the entrainment rate and mixed-layer height prediction from FOJ2 and FOJ3 are similar. The values of $\Theta_{\mathrm{vm}}, U_{\mathrm{m}}, \Delta U, V_{\mathrm{m}}$ and $\Delta V$ from FOJ2 and FOJ3 are also close (Fig. 7c, and e-h), while those from FOJ1 are different from the two other models. Compared to the $\Theta_{\mathrm{vm}}$ values, the $\Delta \Theta_{\mathrm{v}}$ values are less similar to LES data for the same reason discussed in the weak inversion case. Although all of the three models predict $A_{\mathrm{e}}$ reasonably well, all of them underestimate the mixed-layer height. As mentioned above, one possible reason for this is related to the discrepancy of the entrainment heat flux between the first-order jump model and LES. The slight overestimation of $A_{\mathrm{e}}$ may be needed to predict mixed-layer height better. Another reason is associated with quality of inversion layer depth parameterization and uncertainties in the estimation of inversion layer depth from LES data. If inversion layer depth from LES in Fig. 7a is underestimated as mentioned in Pino et al. (2006), the modelled inversion layer depth from FOJ2 and FOJ3 should be regarded as underestimated, which, in turn, underestimates the entrainment rate following Eq. (23). It indicates that the parameterization of the inversion layer depth needs to be improved because the inversion layer depths from the three models can be regarded as underestimated.

In summary, our first-order jump model produces accurate $A_{\mathrm{e}}$ values compared to LES. However, mixed-layer growth and predictions of mixed-layer variables are sensitive to inversion layer parameterizations. When the inversion layer depth is overestimated compared to LES as determined in Pino et al. (2006), the model shows better mixed-layer growth. The evaluation in this section is limited because the LES data are case- and time-limited (total simulation time of 18,000 s) and the comparison of the parameterization has not been made with observations. In a future study, systematic evaluation of the parameterization with extensive observational data (e.g., wind profiler and lidar data) and more LES datasets with different 
surface heat flux and wind shear forcing should be pursued. Our parameterization is not tested against baroclinic CBL cases. Recently, Conzemius and Fedorovich (2004) compared different entrainment flux parameterizations with LES for cases with $S_{x} \neq 0$ and $S_{x}=0$ where $S_{x}$ is the free atmosphere shear in the $x$-direction. They found that both cases produced similar values of the entrainment ratio, and the analyzed parameterizations produce similar results for both cases. However, if one wants to study the performance of the parameterization in a real baroclinic case, it is not sufficient to perform $S_{x} \neq 0$. We need to relate this to a temperature advection as done by Sorbjan (2004). This fact implies that periodic boundary conditions used in the LES simulations are not a proper assumption for the study of a baroclinic CBL.

\section{Conclusions}

The basic entrainment equations applicable to the shear-driven convective boundary layer (CBL) are derived by incorporating a finite inversion depth. By integrating over two distinct layers, i.e., the mixed layer and the inversion layer, we derive an expression of the entrainment heat and momentum fluxes in the framework of the first-order jump model. The parameterization of the entrainment heat flux includes the effect of the inversion layer depth, the convection velocity, the friction velocity, and the velocity jump across the inversion layer. In addition, the inversion layer depth is estimated by the parcel method incorporating velocity jumps across the interfacial layer. Various equations diagnosing the interfacial layer depth are proposed.

The entrainment parameterization developed in this study is further tested with the LES results of two strongly sheared CBLs with different inversion strengths. Compared to the LES data, the first-order jump model described here accurately predicts the evolution of CBL height, $\mathrm{CBL}$ mean temperature, $\mathrm{CBL}$ mean wind components, temperature jump, and velocity jump across the inversion layer. Even though our model determines the entrainment closure ratio correctly, overall mixed-layer growth and predictions of mixed-layer and inversion layer jumps are somewhat sensitive to the parameterization of inversion layer depth. In this study, the inversion layer depth is parameterized with only six available LES datasets. A better parameterization of the inversion layer depth is needed using LES data under various inversion strength and shear conditions.

Acknowledgements This work was supported by the visiting study program of KOSEF (Korea Science and Engineering Foundations), NCAR visiting scientist program, the Stichting Nationale Computerfaciliteiten (National Computing Facilities Foundation, NCF) with the project SG-132 for the use of supercomputing facilities, with financial support from the Netherlandse Organisatie voor Wetenschappelijk Onderzoek (Netherlands Organization for Scientific Research, NWO) and the MCYT scientific project ESP2002-03678. Jordi Vilà was partially supported during his visit to NCAR by the Netherlands Organisation for Scientific Research (NWO) (project R76-241)

\section{Appendix A}

\section{A.1. Derivations of Basic Equations (4)-(6)}

In the following, the derivations of Eqs. (4)-(6) are described. These procedures are applied similarly to the derivations of Eqs. (7)-(12), which are not shown here. The virtual potential temperature profile in the idealized convective boundary layer is givenby 


$$
\Theta_{\mathrm{v}}= \begin{cases}\Theta_{\mathrm{vm}}, & 0 \leq z \leq h_{1} \\ \Theta_{\mathrm{vm}}+\frac{\Delta \Theta_{v}}{\delta}\left(z-h_{1}\right), & h_{1} \leq z \leq h_{2} \\ \Theta_{\mathrm{vm}}+\Delta \Theta_{v}+\gamma\left(z-h_{2}\right), & z \geq h_{2}\end{cases}
$$

where $\Theta_{v}$ is the virtual potential temperature, $\Theta_{v m}$ is the mean mixed-layer virtual potential temperature, $\Delta \Theta_{\mathrm{v}}$ is the virtual potential temperature jump across $h_{1}$ and $h_{2}, h_{1}$ is the height of minimum heat flux or mixed-layer height, $h_{2}$ is the top of boundary layer where the heat flux vanishes, $\delta$ is the distance between $h_{1}$ and $h_{2}$, and $\gamma$ is the vertical virtual potential temperature gradient in the free atmosphere. Equation (4) is obtained by integrating Eq. (1) from 0 to $h_{1}$ and using Leibniz's rule.

$$
\int_{0}^{h_{1}} \frac{\partial \Theta_{\mathrm{v}}}{\partial t} d z=-\int_{0}^{h_{1}} \frac{\partial \overline{w \theta_{\mathrm{v}}}}{\partial z} d z-\int_{0}^{h_{1}} W \frac{\partial \Theta_{\mathrm{v}}}{\partial z} d z,
$$

where $\overline{w \theta_{\mathrm{v}}}$ is the heat flux and $W$ represents the large-scale rising and sinking motions. The left-hand side of (A2) can be rewritten as

$$
\int_{0}^{h_{1}} \frac{\partial \Theta_{\mathrm{v}}}{\partial t} d z=\frac{\partial}{\partial t} \int_{0}^{h_{1}} \Theta_{\mathrm{v}} d z-\Theta_{\mathrm{vm}} \frac{\partial h_{1}}{\partial t}=\frac{\partial}{\partial t}\left(\Theta_{\mathrm{vm}} h_{1}\right)-\Theta_{\mathrm{vm}} \frac{\partial h_{1}}{\partial t}=h_{1} \frac{\partial \Theta_{\mathrm{vm}}}{\partial t} .
$$

Since the vertical virtual potential temperature gradient in the mixed layer is neglected in this idealized temperature profile, the right-hand side of (A2) becomes

$$
-\int_{0}^{h_{1}} \frac{\partial \overline{w \theta_{\mathrm{v}}}}{\partial z} d z-\int_{0}^{h_{1}} W \frac{\partial \Theta_{\mathrm{v}}}{\partial z} d z=\overline{w \theta_{\mathrm{vs}}}-\overline{w \theta_{\mathrm{v}}} h_{1}
$$

where $\overline{w \theta_{\mathrm{vs}}}$ is the surface heat flux and $\overline{w \theta_{\mathrm{v}}} h_{1}$ is the heat flux at $z=h_{1}$. By combining (A3) and (A4), Equation (4) is obtained.

Equation (5) is derived by integrating Equation (2) from $h_{1}$ to $h_{2}$ and using Leibniz's rule.

$$
\int_{h_{1}}^{h_{2}} \frac{\partial \Theta_{\mathrm{v}}}{\partial t} d z=-\int_{h_{1}}^{h_{2}} \frac{\partial \overline{w \theta_{\mathrm{v}}}}{\partial z} d z-\int_{h_{1}}^{h_{2}} W \frac{\partial \Theta_{\mathrm{v}}}{\partial z} d z .
$$

The left-hand side of Eq. (A5) can be rewritten as

$$
\begin{aligned}
\int_{h_{1}}^{h_{2}} \frac{\partial \Theta_{\mathrm{v}}}{\partial t} d z & =\frac{\partial}{\partial t} \int_{h_{1}}^{h_{2}} \Theta_{\mathrm{v}} d z-\left(\Theta_{\mathrm{vm}}+\Delta \Theta_{\mathrm{v}}\right) \frac{\partial h_{2}}{\partial t}+\Theta_{\mathrm{vm}} \frac{\partial h_{1}}{\partial t} \\
& =\frac{\partial}{\partial t}\left(\Theta_{\mathrm{vm}} \delta+\Delta \Theta_{\mathrm{v}} \frac{\delta}{2}\right)-\left(\Theta_{\mathrm{vm}}+\Delta \Theta_{\mathrm{v}}\right) \frac{\partial h_{2}}{\partial t}+\Theta_{\mathrm{vm}} \frac{\partial h_{1}}{\partial t} .
\end{aligned}
$$

The right-hand side of Eq. (A5) is rearranged to

$$
\begin{aligned}
-\int_{h_{1}}^{h_{2}} \frac{\partial \overline{w \theta_{\mathrm{v}}}}{\partial z} d z-\int_{h_{1}}^{h_{2}} W \frac{\partial \Theta_{\mathrm{v}}}{\partial z} d z & =\left.\overline{w \theta_{\mathrm{v}}}\right|_{h_{1}}-\int_{h_{1}}^{h_{2}}(-D z) \frac{\partial \Theta_{\mathrm{v}}}{\partial z} d z \\
& =\left.\overline{w \theta_{\mathrm{v}}}\right|_{h_{1}}+\Delta \Theta_{\mathrm{v}} D\left(h_{1}+\frac{\delta}{2}\right) \\
& =\left.\overline{w \theta_{\mathrm{v}}}\right|_{h_{1}}-\Delta \Theta_{\mathrm{v}} W_{h_{1}+\frac{\delta}{2}} .
\end{aligned}
$$

Here $D(=-\partial W / \partial z)$ denotes the divergence, and is constant with height. With the assumption of $\partial \delta / \partial t=0, W_{h_{1}+\frac{\delta}{2}}=W_{h_{1}}$ and setting (A6) $=(\mathrm{A} 7)$ Eq. (5) is derived. 
Equation (6) is obtained by integrating Eq. (2) from $h_{2}$ to $h_{2}+\varepsilon$, using Leibniz's rule, and applying $\lim _{\varepsilon \rightarrow 0}$ to the integrated equation,

$$
\int_{h_{2}}^{h_{2}+\varepsilon} \frac{\partial \Theta_{\mathrm{v}}}{\partial t} d z=-\int_{h_{2}}^{h_{2}+\varepsilon} \frac{\partial \overline{w \theta_{\mathrm{v}}}}{\partial z} d z-\int_{h_{2}}^{h_{2}+\varepsilon} W \frac{\partial \Theta_{\mathrm{v}}}{\partial z} d z .
$$

The left-hand side of Eq. (A8) is rearranged to

$$
\begin{aligned}
\int_{h_{2}}^{h_{2}+\varepsilon} \frac{\partial \Theta_{\mathrm{v}}}{\partial t} d z & =\frac{\partial}{\partial t} \int_{h_{2}}^{h_{2}+\varepsilon} \Theta_{\mathrm{v}} d z-\left(\Theta_{\mathrm{vm}}+\Delta \Theta_{\mathrm{v}}+\gamma \varepsilon\right) \frac{\partial\left(h_{2}+\varepsilon\right)}{\partial t}+\left(\Theta_{\mathrm{vm}}+\Delta \Theta_{\mathrm{v}}\right) \frac{\partial h_{2}}{\partial t} \\
& =\frac{\partial}{\partial t}\left(\Theta_{\mathrm{vm}} \varepsilon+\Delta \Theta_{\mathrm{v}} \varepsilon+\frac{1}{2} \gamma \varepsilon^{2}\right)-\gamma \varepsilon \frac{\partial h_{2}}{\partial t} .
\end{aligned}
$$

The right-hand side of Eq. (A8) is rewritten as

$$
-\int_{h_{2}}^{h_{2}+\varepsilon} \frac{\partial \overline{w \theta_{\mathrm{v}}}}{\partial z} d z-\int_{h_{2}}^{h_{2}+\varepsilon} W \frac{\partial \Theta_{\mathrm{v}}}{\partial z} d z=\frac{1}{2} \gamma D\left(2 h_{2} \varepsilon+\varepsilon^{2}\right) .
$$

By assuming $\partial \gamma / \partial t=0$, dividing (A9) and (A10) by $\varepsilon$, and applying $\lim _{\varepsilon \rightarrow 0}$ to (A9) and (A10), (A8) becomes

$$
\frac{\partial\left(\Theta_{\mathrm{vm}}+\Delta \Theta_{\mathrm{v}}\right)}{\partial t}=\gamma\left(\frac{\partial h_{2}}{\partial t}+D h_{2}\right)=\gamma\left(\frac{\partial h_{2}}{\partial t}-W_{h_{2}}\right) .
$$

With the assumptions of $\partial \delta / \partial t=0$ and $W_{h_{2}}=W_{h_{1}}$, (A11) is equal to Eq. (6).

\section{Appendix B}

\section{B.1. Derivations of Eq. (19)}

Below are the derivations of Eq. (19),

$$
\begin{aligned}
\int_{0}^{h_{2}}\left[\overline{u w} \frac{\partial U}{\partial z}+\overline{v w} \frac{\partial V}{\partial z}\right] d z= & \int_{0}^{h_{\mathrm{s}}}\left[\overline{u w} \frac{\partial U}{\partial z}+\overline{v w} \frac{\partial V}{\partial z}\right] d z+\int_{h_{\mathrm{s}}}^{h_{1}}\left[\overline{u w} \frac{\partial U}{\partial z}+\overline{v w} \frac{\partial V}{\partial z}\right] d z \\
& +\int_{h_{1}}^{h_{2}}\left[\overline{u w} \frac{\partial U}{\partial z}+\overline{v w} \frac{\partial V}{\partial z}\right] d z .
\end{aligned}
$$

The first term on the right-hand side (rhs) in (B1) is integrated such that

$$
\int_{0}^{h_{\mathrm{s}}}\left[\overline{u w} \frac{\partial U}{\partial z}+\overline{v w} \frac{\partial V}{\partial z}\right] d z=\overline{u w}_{\mathrm{s}} U_{\mathrm{m}}+\overline{v w}_{\mathrm{s}} V_{\mathrm{m}}=-C_{\mathrm{D}}^{-1 / 2} u_{*}^{3} .
$$

To derive Eq. (B2), the following relationships are used: $\overline{u w}_{\mathrm{s}}=-C_{\mathrm{D}} M U_{\mathrm{m}}, \overline{v w}_{\mathrm{s}}=$ $-C_{\mathrm{D}} M V_{\mathrm{m}}, M=\sqrt{U_{\mathrm{m}}^{2}+V_{\mathrm{m}}^{2}}, u_{*}^{2}=\sqrt{\left({\overline{u w_{\mathrm{s}}}}^{2}+\left({\overline{v w_{\mathrm{s}}}}^{2}\right.\right.}=C_{\mathrm{D}} M^{2}$ (Stull, 1988).

Since the gradient of wind in the mixed layer can be neglected, the second term on the rhs in $(\mathrm{B} 1)$ is

$$
\int_{h_{\mathrm{s}}}^{h_{1}}\left[\overline{u w} \frac{\partial U}{\partial z}+\overline{v w} \frac{\partial V}{\partial z}\right] d z=0
$$


The third term on the rhs in (B1) is integrated such that

$$
\int_{h_{1}}^{h_{2}}\left[\overline{u w} \frac{\partial U}{\partial z}+\overline{v w} \frac{\partial V}{\partial z}\right] d z=\frac{1}{2} \overline{u w}_{h_{1}} \Delta U+\frac{1}{2} \overline{v w}_{h_{1}} \Delta V .
$$

To derive Eq. (B4), the following relationships are used: $\overline{u w}=-\frac{\overline{u w}_{h_{1}}}{\delta}\left(z-h_{1}\right)+\overline{u w}_{h_{1}}$, $\overline{v w}=-\frac{\overline{v w}_{h_{1}}}{\delta}\left(z-h_{1}\right)+\overline{v w}_{h_{1}}, \frac{\partial U}{\partial z}=\frac{\Delta U}{\delta}, \frac{\partial V}{\partial z}=\frac{\Delta V}{\delta}$. Equation (B4) is arranged such as

$$
\frac{1}{2} \overline{u w}_{h_{1}} \Delta U+\frac{1}{2} \overline{v w}_{h_{1}} \Delta V=\frac{-\delta u_{*}^{2}\left(\frac{U}{M} \Delta U+\frac{V}{M} \Delta V\right)-2 h_{1} w_{\mathrm{e}}(\Delta \tilde{U})^{2}}{4 h_{1}+2 \delta} .
$$

To derive Eq. (B5), Equations (14) and (15), $\overline{u w}_{\mathrm{s}}=-C_{\mathrm{D}} M U_{\mathrm{m}}, \overline{v w}_{\mathrm{s}}=-C_{\mathrm{D}} M V_{\mathrm{m}}$, $M=\sqrt{U_{\mathrm{m}}^{2}+V_{\mathrm{m}}^{2}}$, and $u_{*}^{2}=\sqrt{\left({\overline{u w_{\mathrm{s}}}}^{2}+\left({\overline{v w_{\mathrm{s}}}}^{2}\right.\right.}=C_{\mathrm{D}} M^{2}$ are used.

\section{References}

Ayotte KW, et al. (1996) An evaluation of neutral and convective planetary boundary layer parameterizations relative to large eddy simulations. Boundary-Layer Meteorol 79:131-175

Betts AK (1973) Non-precipitating cumulus convection and its parameterization. Quart J Roy Meteorol Soc 99:178-196

Betts AK, Barr AG (1996) First international satellite land surface climatology field experiment 1987 sonde budget revisited. J Geophys Res 101:23,285-23,288

Boers R, Eloranta EW, Coulter RL (1984) Lidar observations of mixed layer dynamics: Tests of parameterized entrainment models of mixed layer growth rate. J Clim Appl Meteorol 23:247-266

Conzemius R, Fedorovich E (2004) Numerical models of entrainment into sheared convective boundary layers evaluated through large eddy simulations. Proceedings of the $16^{\text {th }}$ conference on boundary layer and turbulence. American Meteorological Society

Cuijpers JWM, Holtslag AAM (1998) Impact of skewness and nonlocal effect on scalar and buoyancy fluxes in convective boundary layers. J Atmos Sci 55:151-162

Deardorff JW (1972) Parameterization of the planetary boundary layer for use in general circulation models. Mon Wea Rev 100:93-106

Deardorff JW (1979) Prediction of convective mixed-layer entrainment for realistic capping inversion structure. J Atmos Sci 36:424-436

Driedonks AGM (1982) Models and observations of the growth of the atmospheric boundary layer. Boundary-Layer Meteorol 23:283-306

Driedonks AGM (1984) Entrainment effects in the well-mixed atmospheric boundary layer. Boundary-Layer Meteorol 30:75-105

Fedorovich E, Mironov DV (1995) A model for a shear-free convective boundary layer with parameterized capping inversion structure. J Atmos Sci 52:83-95

Fedorovich E, Nieuwstadt FTM, Kaiser R (2001) Numerical and laboratory study of horizontally evolving convective boundary layer. Part II : Effects of elevated wind shear and surface roughness. J Atmos Sci 58:546-560

Fedorovich E, Conzemius R, Esau I, Chow FK, Lewellen D, Moeng C-H, Sullivan P, Pino D, Vilà-Guerau de Arellano J (2004) Entrainment into sheared convective boundary layers as predicted by different large eddy simulation codes. Proceedings of the 16th conference on boundary layer and turbulence, American Meteorological Society.

Flamant C, Pelon J (1996) Atmospheric boundary layer structure over the Mediterranean during a Tramontane event. Quart J Roy Meteorol Soc 122:1741-1778

Flamant C, Pelon J, Flamant PH, Durand P (1997) Lidar determination of the entrainment zone thickness at the top of the unstable marine atmospheric boundary layer. Boundary-Layer Meteorol 83:247-284

Flamant C, Pelon J, Brashers B, Brown RA (1999) Evidence of a mixed-layer dynamics contribution to the entrainment process. Boundary-Layer Meteorol 93:47-73

Gryning S, Batchvarova E (1990) Analytical model for the growth of the coastal internal boundary layer during a offshore flow. Quart J Roy Meteorol Soc 116:187-203

Kim S-W, Park S-U, Moeng C-H (2003) Entrainment processes in atmospheric boundary layer structure driven by Wind Shear and Surface Heat Flux. Boundary-Layer Meteorol 108:221-245 
Klemp JB, Durran DR (1983) An upper boundary condition permitting internal gravity wave radiation in numerical mesoscale models. Mon Wea Rev 111:430-444

Moeng C-H (1984) A large-eddy simulation model for the study of planetary boundary-layer turbulence. J Atmos Sci 41:2052-2062

Moeng C-H, Sullivan PP (1994) A comparison of shear and buoyancy driven planetary boundary layer flows. J Atmos Sci 51:999-1022

Pino D, Vilà-Guerau de Arellano J, Duynkerke PG (2003) The contribution of shear to the evolution of a convective boundary layer. J Atmos Sci 60:1913-1926

Pino D, Vilà-Guerau de Arellano J, Kim S-W (2006) Representing sheared convective boundary layer by zeroth- and first-order jump mixed layer models: Large-eddy simulation verification. J Appl Meteorol (In press)

Randall DA (1984) Buoyant production and consumption of turbulent kinetic energy in cloud-topped mixed layers. J Atmos Sci 41:402-413

Spalart PR, Moser RD, Rogers MM (1991) Spectral methods for the Navier-Stokes equations with in infinite and two periodic directions. J Comp Phys 96, 297

Sorbjan Z (2004) Large-eddy simulations of the baroclinic boundary layer. Boundary-Layer Meteorol 112: $57-80$

Stull RB (1988) An introduction to boundary layer meteorology. Kluwer Academic Publishers, Dordrecht $666 \mathrm{pp}$

Suarez MJ, Arakawa A, Randall DA (1983) Parameterization of the planetary boundary layer in the UCLA general circulation model: formulation and results. Mon Wea Rev 111:2224-2243

Sullivan PP, McWilliams JC, Moeng C-H (1994) A subgrid-scale model for large-eddy simulation of planetary boundary-layer flows. Boundary-Layer Meteorol 71:247-276

Sullivan PP, McWilliams JC, Moeng C-H (1996) A grid nesting method for large eddy simulation of planetary boundary-layer flows. Boundary-Layer Meteorol 80:167-202

Tennekes H (1973) A model for the dynamics of the inversion above a convective boundary layer. J Atmos Sci 30:558-567

Tennekes H, Driedonks AGM (1981) Basic entrainment equations for the atmospheric boundary layer. Boundary-Layer Meteorol 20:515-531

van Zanten MC, Duynkerke PG, Cuijpers JWM (1999) Entrainment parameterization in convective boundary layers. J Atmos Sci 56:813-828

Zeman O, Tennekes H (1977) Parameterization of the turbulent energy budget at the top of the daytime atmospheric boundary layer. J Atmos Sci 34:111-123 